\title{
Pragmatic Considerations in the Interpretation of Denying the Antecedent ${ }^{1}$
}

\author{
ANDREI MOLDOVAN
}

Philosophy Department

University of Barcelona

Montalegre 6, $4^{\mathrm{a}}$ planta, Barcelona

Spain

mandreius@yahoo.com

\begin{abstract}
In this paper I am concerned with the analysis of fragments of a discourse or text that express arguments suspected of being denials of the antecedent. I first argue that one needs to distinguish between two senses of 'the argument expressed'. Second, I show that, with respect to one of these senses, given a Gricean account of the pragmatics of conditionals, some such fragments systematically express arguments that are valid.
\end{abstract}

Résumé: Dans cet article je concentre mon attention sure une analyse de fragments de discours ou de texte qui expriment des arguments dans lesquels on semble nier l'antécédent d'une proposition conditionnelle. J'avance premièrement qu'on doit distinguer deux significations de « l'argument exprimé ». Ensuite je montre que par rapport à une de ces significations, étant donné l'explication pragmatique des propositions conditionnelles de Grice, certains de ces fragments expriment systématiquement des arguments valides.

Keywords: conditional perfection, denying the antecedent, fallacy, Grice, implicature, rational reconstruction

\section{Introduction}

In this paper I am concerned with the analysis ${ }^{2}$ of fragments of discourse or text (utterances, in general), which express arguments suspected of being denials of the antecedent (DA from now on). I will focus on pragmatic aspects of argument analysis with respect

\footnotetext{
${ }^{1}$ This paper was awarded the J. Anthony Blair Prize for an outstanding paper by a student at the Ontario Society for the Study of Argumentation conference, Argument Cultures, held at the University of Windsor, Windsor, Ontario, Canada on 3-6 June 2009. - The Editors.

${ }^{2}$ See Ralph Johnson (2000 p.40) for the distinction between two aspects of the study of argument: the descriptive and the evaluative one. The former is the subject matter of the Theory of Analysis, the latter, of the Theory of Appraisal.
}

(C) Andrei Moldovan. Informal Logic, Vol. 29, No. 3 (2009), pp. 309-326. 
to the identification of the premises of an argument. I will first argue that one needs to distinguish between two senses of 'the argument expressed'. Second, I will show that, with respect to one of these senses, some such fragments express arguments that are valid, and do not instantiate DA. I will appeal to a Gricean accountof the pragmatics of conditionals in order to support my conclusion. Finally, I will discuss and reject an objection to my thesis.

\section{The fallacy of denying the antecedent}

An argument is materially valid (or deductively valid, as some people prefer to say) if and only if it is impossible that its premises are all true and its conclusion is false. Some arguments instantiate the fallacy of denying the antecedent, which is to say that they have the form: If $p, q$. $\neg p$. Therefore, $\neg q$. The arguments that instantiate this form are materially (i.e. deductively) invalid. There are, however, exceptions: some of the arguments that have this form are materially valid, due to semantic entailments, or due to the fact that they instantiate another valid logical form. ${ }^{3}$

Material validity is defined in terms of truth. So, given that the arguments that instantiate DA can be evaluated for validity, they must have structural elements that are capable of bearing a truthvalue. I will take these elements, such as $p$ and $q$ above, to be propositions. ${ }^{4}$ So, I will assume that the logical structure of such an argument contains a set of propositions $\mathrm{P}=\{p 1, \ldots p n, c\}$, where $p l, \ldots p n$ are the premises and $c$ the conclusion of the argument. Identifying this set of propositions is identifying the logical structure of the argument. This is necessary for evaluating the argument for its validity. It is not meant to be a definition of 'deductive arguments', but rather as a useful tool for reconstructing and evaluating certain arguments. However, this characterization of the logical structure of an argument is compatible with defining deductive argument in terms of criteria most proper for their evaluation, as Erik Krabbe does. He writes that by 'deductive arguments' he means "(single) arguments that invite an evaluation in terms of deductive criteria, even though they may not exclude the use of other criteria" (Krabbe 2003, p. 1).

\footnotetext{
${ }^{3}$ See Godden \& Walton (2004, p. 222) for examples and a discussion of such cases.

${ }^{4}$ Johnson (2000, p. 168) defines argument in terms that make no reference to propositions, premises or conclusion. Other authors are less radical in eschewing talk of propositions. Robert Pinto writes: "A set of propositions constitutes a set of premises and a conclusion $p$ if and only if someone puts them forward as premises for $p$ in the course of arguing for $p$ " (Pinto, 2001 p. 1).
} 
The Theory of Analysis deals with the problem of how to identify and reconstruct an argument that is put forward in a text or oral discourse. Its aim is then that of interpreting a fragment of a text or discourse or a contribution to a dialog. With respect to the issue of evaluation arguments for their validity, the problem comes down to identifying, among all the propositions a fragment of text or discourse conveys, the propositions that constitute the set $\mathrm{P}$ of the argument.

\section{Pragmatic aspects of interpretation}

In order to approach the problem of how to identify P, I will mention briefly some points that have been traditionally made about the interpretation of any discourse or text, be it argumentative or not.

First of all, one useful concept is that of what is said, a concept introduced by H.P. Grice (1989, p. 25). This is, roughly speaking, the proposition literally expressed by the sentence uttered. Grice characterizes it as "closely related to the conventional meaning of the words (the sentence) [the speaker] has uttered" (Grice 1989, p. 25 ). What is said is closely related but not identical to conventional meaning because in many cases, apart from the literal meaning of the words uttered, contextual information is needed for the interpretation to deliver a propositional content at all. Contextual information is relevant in interpreting indexicals, demonstratives, ambiguous expressions, as well as in ellipsis and anaphora resolution.

The context also plays an essential role in getting at other propositions that speakers communicate apart form what is said by their utterances. Speakers may make use of irony, suggestion, metaphor, presupposition, and so on. In these cases speakers mean more than what they say. Grice uses the term 'implicatures' to refer to these propositions, which always differ from what is said, but may be entailed, or merely suggested by it. Conversational implicatures are those implicatures that depend heavily on the details of the context in which an utterance is made. With respect to these implicatures, Grice argues that they are always derivable, which means that an audience should only interpret a speaker as implicating a proposition if she is in the position to infer the proposition implicated from what is said, together with other information available in the context of utterance, and the assumption that the speaker acts according to the conversational maxims of rational and cooperative behaviour. Grice writes:

The final test for the presence of a conversational implicatures has to be, as far as I can see, a derivation of it. One has to produce an account of how it could have 
arisen and why it is there. And I am very much opposed to any kind of sloppy use of this philosophical tool, in which one does not fulfil this condition. (Grice, quoted in Neale 1990, p. 78)

Stephen Neale refers to the requirement that Grice places on interpreters as the Justification Requirement (Neale 1990, p. 78). The inferential schema behind the requirement can be summarized as follows: a hearer is justified in taking a speaker to conversationally implicate that proposition which the speaker must be assumed to believe in order to preserve the assumption that the speaker is adhering to the conversational maxims. In order to avoid violations of conversational maxims, the speaker must be taken to intend to communicate more than what is said by her utterance.

As Deirdre Wilson and Dan Sperber make clear, the inference behind the Justification Requirement "plays little if any role in the recovery of implicatures" (Wilson\&Sperber 1986, p. 378). That is, getting at what proposition a speaker implicates is a question of hypothesis formation, which is usually dealt with intuitively by interpreters. It was not among Grice's aims to clarify this process. His aim was rational reconstruction of speaker's communicative intentions. The Justification Requirement plays an essential role in the latter, but not in the former. I will come back to this point later.

\section{Two concepts of 'the argument expressed'}

Rational reconstruction of what is implicit in a text or discourse that is suspected of expressing an argument is relevant to the Theory of Analysis of arguments, given that utterances need to be interpreted in order for the arguments to be identified. Explicitness is an important value in argumentation, as well as in communication in general. ${ }^{5}$ But there is no reason to restrict the interpretation of a text or discourse merely to what is explicitly stated, excluding implicatures or presuppositions from the interpretation. Suppose we restrict the interpretation only to what is said by each of the utterances of the discourse or text. Appealing to argument indicators, as well as to meta-discursive indications (if available) about the speaker's intentions and purposes, we would then obtain a set $\mathrm{P}$ of propositions, one of which is the conclusion, the other the premises of an argument. Let us call the argument that has this structure argument-w (' $\mathrm{w}$ ' from what is said). Similar considerations, but this time taking into account presuppositions and implicatures as well, which are not literally expressed, would lead to what we can call the argument-m (' $\mathrm{m}$ ' from meant)

\footnotetext{
${ }^{5}$ For the value of explicitness for argumentation, see Adler (2002, pp. 86-91)
} 
conveyed. So 'the argument expressed' can be understood in at least two senses: the argument literally expressed by the sentences uttered; and the argument the speaker meant, which includes in $\mathrm{P}$ implicatures and presuppositions.

Whether it is more important to focus on argument-w or on argument-m is a question that I do not want to settle here. I do not even know whether it has one answer, or whether the answer depends on our purposes as evaluators. What I claim is that, in as much as reconstructing the argument-m that a speaker conveys is important, attention should be paid to pragmatic elements involved, and especially to implicatures.

\section{Conditional perfection}

In what follows I will focus on one kind of implicature that has been studied extensively, and which serves to interpret certain linguistic phenomena. What is usually called 'Conditional Perfection' (CP) is a phenomenon that consists in treating an utterance of 'If $p$ then $q$ ' as expressing not only that $p$ is a sufficient condition for $q$, but also that it is a necessary condition. That is to say, people tend to treat 'If $p$ then $q$ ' as expressing if and only if $p$, then $q$. This phenomenon is independent of whether the context is one in which arguments are given and evaluated, or not. For example, when a father says to his son 'If you mow the lawn, I will give you five dollars' he may be taken to have asserted that only if the kid mows the lawn will he give him $\$ 5$. One of the first to have observed this phenomenon was Oswald Ducrot (1969), followed by M. Geis and A. Zwicky (1971), who rediscovered it. ${ }^{6}$ In the terminology of the latter, the utterance of 'If $p$ (then) $q$ ' suggests or invites the inference to $q$, only if $p$, which can be better expressed as if not-p then not-q.

Most authors have argued that conditional perfection is to be explained as an essentially linguistic phenomenon. Instead of taking 'if' as lexically ambiguous, largely for the reasons that Grice (1989, pp. 47-49) put forward against multiplying senses by postulating ambiguities, some authors argue that the literal meaning of 'if' be given by the truth-functional analysis. That is, the antecedent of a conditional introduces a sufficient condition for the consequent to be the case. At the same time, they offer a pragmatic explanation of CP. The phenomenon is usually treated as involving pragmatic strengthening of the content of the utterance,

\footnotetext{
${ }^{6}$ For a history of the successive rediscoveries of CP, see van der Auwera (1997).

${ }^{7}$ Horn (2000) points out that it has been observed that ' $q$ only if $p$ ' is better paraphrased by 'If not- $p$ then not- $q$ ' (which is the inverse of the conditional) than by 'If $q$ then $p$ ', or by ' $p$ if $q$ ', at least when $p$ and $q$ have different temporal and causal implications. I will follow this suggestion.
} 
in the sense that the invited inference is to be explained as an implicature. However, not all authors agree on the details of the explanation.

Geis and Zwicky argue that, "what we have called 'invited inferences' constitutes a special class of 'implicatures', although they are clearly distinct from the "conversational implicatures" (Geis \& Zwicky 1971, p. 5). More recent authors, such as J. van der Auwera (1997) and L. Horn (2000), consider that CP is due to a scalar conversational implicature that is triggered by the utterance of the conditional. However, they differ in their account of the scalar implicature. Van der Auwera considers the scale $\mathrm{S}$ of propositions as being the one involved in deriving the implicature.

(S) $\ldots$

if $p, q$ and if $r, q$ and if $s, q$

if $p, q$ and if $r, q$

if $p, q$

The proposition at the bottom constitutes what is said by father's utterance, i.e. it is the proposition that has been asserted. The higher propositions in the scale are conditionals whose antecedents express possible sufficient conditions for $q$ to be the case. The upper propositions entail the lower ones, and so the upper ones are more informative. Van der Auwera explains the Gricean derivation of the implicature that $p$ is a necessary condition as follows:

Standard scalar implicatures arise as negations of the higher assertions, and this is also what we find here... when one supplies only the one sufficient condition $p$, one conversationally implicates that there is no second-and no third, etc. - sufficient condition. (van der Auwera 1997, p. 262.)

Given the presumption that the speaker observes the maxim of Quantity (in particular, the first submaxim, which requires that the speaker make his contribution as informative as is needed for purposes of the exchange), and given his utterance of 'if $p, q$ ', the audience is in the position to infer that $r$ or $s$ are not sufficient conditions for the truth of $q$. If $r$ and $s$ had been sufficient conditions for $q$, the speaker would have violated the maxim by not mentioning them. This way the audience will rule out all other sufficient condition except $p$, and conclude that $p$ is a necessary condition as well. So, the implicature is a negation of higher propositions in the scale. The only sufficient condition becomes also a necessary one, and the conditional is strengthened to a biconditional. 
It is not my purpose in this paper to defend this account of CP. However, for my own purposes I need to present it in a light that makes it sufficiently convincing. So I will take some time now to defend it from some objections that may immediately come to mind. Dealing with these objections will help clarify the nuances of the account under consideration.

One objection is voiced by L. Horn, who writes that in van der Auwera's account the propositions that appear higher in the scale "never seem to figure directly in the reasoning that takes us from if $p$ then $q$ to if not-p then not- $q$ " (Horn 2000, p. 305). But the version of this account of CP that I propose does not require the audience to entertain a potentially infinite scale of conjunctions of conditional propositions. Actually, the audience is not even required to entertain any propositions in the scale apart from the one at the bottom. It is merely the possibility that there be other conditionals with $q$ as a consequent and a contextually relevant antecedent that the audience needs to take into account. The audience may rule out any candidate for a further sufficient condition she envisages by thinking of it as appearing in the scale; but she is not required to entertain any such conditional in order to rule them all out. That is to say, the scale $\mathrm{S}$ presented above may be misleading in various ways. It is here rather for illustrative purposes, because what is central in the derivation of scalar implicatures is the maxim of Quantity.

One important observation, which will help deal with an objection later, is that at the bottom of the scale there need not be a simple conditional, as in the scale $\mathrm{S}$ presented above. The bottom of the scale may be something like 'if $p, q$ and if $r, q$ ', or even a longer conjunction of conditionals. The scalar implicature is then that there are no other sufficient conditions (apart from $p$ and $r$, respectively). In this case there is no implicature that any condition is necessary, as both $p$ and $r$ are sufficient, but none is necessary. So there is no strengthening of a conditional to a bi-conditional. For example, an inscription in a bus may read: 'You are allowed to sit in this seat if you are disabled or older than 70.' Given that on a truth-functional account of conditionals, 'if $p$ or $r$, then $q$ ' is truthfunctionally equivalent to 'if $p, q$ and if $r, q$ ', the bottom line of the scale will be in this case not a simple conditional but a conjunctions of conditionals. The implicature is that there is no other sufficient condition for being allowed to occupy the seat. The conditionals that constitute the bottom of the scale need not be stated in the same sentence, but at different places of a text or discourse. Or they may be available to the participants in the conversation as part of the common background assumptions, and not be stated anywhere in the conversation.

This observation helps deal with the objection that the scalar account presented over-generates implicatures, in the sense that it 
will strengthen any conditional statement to a bi-conditional. And, the objection goes, there are clear cases of conditionals that convey merely sufficient conditions, and not necessary ones. While I think this last observation is correct, it is not true that the present account predicts that any assertion of a conditional will be strengthened to a bi-conditional. Imagine the explicit statement of the rules of a certain game. There one can find different sufficient conditions for a player to be penalized, which may be stated at different points in the text. In that case the scale will have at the bottom line the conjunction of all the conditionals that state sufficient conditions, and the implicature will be that there are no others. No implicature is derivable before the list, the enumeration, or the conjunction of conditionals is complete. That is, the implicature that there are no other sufficient conditions for penalization will only be derivable when the speaker or writer puts an end to the fragment of discourse or text in which the conditions are conveyed (of course, when there is no explicit mention that the list is complete, in which case there is no implicature to that effect).

It has been argued that a problem for van der Auwera's scalar implicature rests on the fact that assertions that are higher in the scale require more effort to utter. Consequently, there are considerations of economy that suffice to motivate the speaker not to give the whole set of sufficient conditions, even in cases in which she believes that there are more than the one (or the ones) explicitly stated. Therefore, it is wrong to take the speaker to have implicated that there are no other sufficient conditions. ${ }^{8}$ I think one could reply to this objection along the following lines: there is a maxim of Manner that requires of speakers to be brief; but there is no requirement to regard considerations of economy as more important than that of being sufficiently informative for the purpose of the conversation. On the contrary, Grice writes that "It is obvious that the observance of some of these maxims is a matter of less urgency than is the observance of others; a man who has expressed himself with undue prolixity would, in general, be open to milder comment than would a man who has said something he believes to be false" (Grice 1989, p. 27). In the same vein, it is reasonable to think that the first maxim of Quantity is also more

\footnotetext{
${ }^{8}$ Such considerations of economy are at the root of Horn's criticism of van der Auwera's account of the scalar implicature. Horn writes that an utterance of W "will Q(uantity)-implicate that the speaker was not in a position to affirm ... S ... (where ... S ... is informationally stronger than, i.e. unilaterally entails ...W ...) only if $\mathrm{S}$ is at least as lexicalized as $\mathrm{W}$ within the relevant domain... But when $\mathrm{S}$ is less economical than $\mathrm{W}$ in the appropriate sense, no Q-implicature is triggered even when unilateral entailment provides the necessary strength differential." (Horn 2000 p.306-7) And later: "pace van der Auwera, Manner does matter." (idem p.308) He makes reference here to the third maxim of Manner: "Be brief (and avoid unnecessary prolixity)." (Grice 1989 p.27)
} 
important than the requirement to be brief and avoid prolixity. If this is so, and given that cooperative behaviour entails observing the maxims and their order of importance, a cooperative speaker will not leave out information that is required for the purpose of the conversation for reasons having to do only with economy of effort. Of course, a certain degree of idealization is involved in assuming that speakers do observe the maxims and their order of importance. It may be that speakers violate some of the maxims or the order of their importance more or less frequently, and so, that the idealization is not realistic. But within the limits of the Gricean idealization, speakers convey all the information that is needed for the purpose of the conversation and no more, as the maxim of Quantity requires.

A different objection to this account goes as follows: in order to be cooperative the speaker must be as informative as required for the purpose of the conversation. Now, if the speaker has only stated one sufficient condition, it may not be because she believes it is the only sufficient condition. It may simply be that in certain contexts the sufficient condition stated is all that is needed to be as informative as required for the purpose of the conversation. The other conditions may simply not be relevant for the purpose of the conversation. But not being relevant for the purpose of the conversation is different from not being a sufficient condition at all. If the bus driver tells a child: 'You are allowed to sit on that chair if you have a disability,' the driver should not be taken to intend to mean that the only sufficient condition for sitting in the chair is having a disability; she knows very well that it is not. It is merely the only one relevant in the context, as the child is obviously not older than 70 .

I want to concede that this is indeed a powerful objection with respect to the account that has been sketched so far. The scalar account presented rules out all candidates for other sufficient conditions apart from the one actually mentioned on the basis that they would have been asserted if they were indeed sufficient. But a speaker who observes the conversational maxims (in particular, the maxim of relevance) will not mention anyway a sufficient condition that she knowns (or believes) not to be fulfilled. In the case under consideration, given that the condition related to age is known not to be fulfilled, the bus driver will not have mentioned it anyway. So, such a condition will not figure in the upper part of the scale, i.e., among the conditions that would have been asserted if they were sufficient. Therefore, this condition will not be ruled out by the scalar account of the implicature presented so far. However, it is reasonable to think that the driver does implicate that having a disability is the only condition that the child might fulfill in order to use the seat. What needs to be added to van der Auwera's initial proposal is that the condition that relates to the age is ruled out not 
by the scalar implicature, but for reasons of irrelevance: given that the speaker knows that the child is younger than 70 , the condition related to age will not be relevant in the context of the conversation; but the same does not apply for the condition related to disability, as the child might have one that the driver is not aware of. For reasons of relevance, the speaker can be assumed to have ruled out all the sufficient conditions that she know (or believes) the relevant agent (the child, in our example) does not satisfy. This way, the work done by the maxim of Relation (Be relevant) adds to the work done by the maxim of Quantity. The bus driver conveys the implicature that having a disability is the only condition relevant in the context, in the sense that it is the only one that the child might fulfill (in the sense that it is not compatible with what the speaker knows that the child fulfills any other condition). In conclusion, the speaker conveys that it is necessary for the child to fulfill this condition in order to be allowed to use the seat.

\section{CP and DA arguments}

Keeping in mind these modifications of van der Auwera's account of the scalar implicature involved in $\mathrm{CP}$, let us go back to the issue of interpreting utterances that express arguments of DA form. Consider a child who asks his father if she can go play basketball, to which the father answers: 'If you finish your homework you are allowed to play basketball.' Later on, the child tells her friend on the phone that she will not be able to go out

(1) 'If I finish my homework, my dad will let me play basketball. But I will not finish it today; it's just too difficult. So, he will not let me play basketball today.'

The presence of 'so' in the child's utterance indicates that the child intends to put forward an argument. Consider the distinction made above between the argument- $w$ and the argument- $m$ that a fragment of discourse may express. The argument-w expressed by her utterance has (roughly) the form: if $H, P$. $\neg H$. Therefore, $\neg P$. So the argument-w instantiates a DA form and is deductively invalid. Indeed, the premises can be all true while the conclusion false, as the conditional only states a sufficient condition, not a necessary one.

Let us consider now the argument-m. Taking into consideration the account of CP presented above and the father's utterance, it is reasonable to think that doing the homework is a necessary condition in the context for the child to be allowed to go and play. It is not something the father said, but his assertion 
invites the child to perfect the conditional. If there were other sufficient conditions, given the child's manifestation of her interest to go out and play basketball, it would have been cooperative for the father to assert them. Scale $\mathrm{S}$ can be used to derive the implicature that there are no other sufficient conditions relevant in the context, and so that the condition of finishing the homework is necessary for the truth of the consequent. If cleaning her room, mowing the lawn, or taking the dog for a walk were sufficient conditions (which play the role of $r$ and $s$ in the scale) the father is expected to have mentioned them, in as much as he is presumed to observe the first submaxim of Quantity and the maxim of Relation. Under this assumption it is rational to derive the implicature that the inverse of the conditional also holds. Therefore, the father's utterance conveys a necessary condition for the truth of the consequent, not merely a sufficient one. For the same reason the implicature is also present in the child's statement of the conditional. Therefore, even if the argument-w, which results from considering what the child says, is invalid, the argument-m, that includes the implicature as well, is valid. If the implicature is that only if I finish my homework, my dad will let me play basketball, that is, if I do not finish my homework, my dad will not let me play basketball, the argument-m has the form: if $\neg H, \neg P$. $\neg H$. So, $\neg P$. This is a valid modus ponens argument. Of course, the argument-m also has the premise explicitly stated, if $H, P$. But this premise does not influence the validity of the argument.

Whether this argument is the argument the speaker expressed, or whether it is the only one that is of interest for the purpose of argument evaluation, are further question that I have no intention to settle now. However, I take it that there are strong reasons to think that the argument-m is of certain interest, reasons which have to do with the general interpretative strategies of speaker-meaning that I mentioned in Section 2. Given that conditional perfection is in this case justified, in a certain sense, a sense related to argument-m, no fallacy has been committed. ${ }^{9}$ This conclusion is compatible with the claim that the tendency to perfect the conditional "is manifested in two classes of logical fallacies, Affirming the Consequent... and Denying the Antecedent..." (Geis \& Zwicky 1971, p. 2). Once the

\footnotetext{
${ }^{9}$ One could say that in this case the argument superficially is of a DA form, but actually it is a modus ponens. David Hitchcock analyses a fragment of text somewhat similar to (1) and writes: "there is a valid form of argument, which can superficially look like the predicate-logic analogue of denying the antecedent" (Hitchcock 1995), although it is not of that form, according to his interpretation of the text. However, I want to avoid talking about the argument expressed. It is not clear to me that we should always focus on the argument-m, and that this is the argument expressed. It may be useful in a context of argumentation to focus on the argument-w, and consequently to attribute fallacy to the speaker. This may contribute to enhancing explicitness.
} 
distinction between argument- $\mathrm{w}$ and argument-m is kept in mind there is no reason to think that no fallacy was committed at all.

\section{Some clarifications}

A few clarifications are needed at this point. First, my reason for treating the child's utterance as not expressing a fallacious argument-m differs from other reasons that have been given in the literature for a similar conclusion. Thus, Michael Burke (1994) argues in favor of interpreting utterances similar to (1) as not expressing a fallacious argument. His claim is that a non-fallacious interpretation is always preferable "unless the balance of textual, contextual, and other evidence" (Burke 1994 p.24) favors the fallacious interpretation. And so, he suggests that one should take the conditional as not being asserted with the intention of making it a premise in the argument, but only for rhetorical or dialectical reasons. He holds that this interpretation should be preferred on the grounds of a weak charity principle (what he calls 'fairness' in interpretation). D. Godden and D. Walton reply that in such cases "there is a very good reason to suppose that the stated conditional claim is part of the argument: namely, that it is stated" (Godden \& Walton 2004, p. 226). My reasons for rejecting fallacy attribution in (1) have nothing to do with charity considerations. I have not argued that the speaker must have meant by the conditional a biconditional just for the reason of avoiding attributing a fallacy to the speaker. Instead, I have offered a Gricean justification for believing that the inverse of the conditional has also been conveyed. ${ }^{10}$ If it has been conveyed by the fragment of discourse that contains the argument, we should treat is as part of the argument.

Second, I want to point out that my account does not generalize to all instances of DA argument-w's. Suppose someone utters (2), as in Aristotle's famous example, with the purpose of giving an argument:

(2) 'If it has just rained the streets are wet. It has not rained. So, the streets are not wet.'

The Gricean account of CP appealed to so far does not yield the result that the speaker implicates that there are no other sufficient conditions for the streets to be wet apart from rain, i.e., the inverse

${ }^{10}$ Such Gricean considerations show that Wesley Salmon was right in his comments on an example similar to (1): "Actually, people often say "if" when they mean "if and only if"; if the first premise is construed in that way, the argument, of course, becomes valid, though it loses some of its rhetorical force." (Salmon, 1984, my emphasis) 
of the conditional in (2). The hearer, and even the speaker, may be tempted to perfect the conditional and conclude that there are no other sufficient conditions apart from rain. They might construe a scale $\mathrm{S}$ of propositions (in which $r$ and $s$ are conditions such as the cleaning of streets with water, a river flood, etc.), and perfect the conditional under the assumption that the speaker observes the first submaxim of Quantity and the maxim of Relation. The scalar implicature will be a denial of upper possible assertions in the scale to the effect that the speaker believes that $r$ and $s$ are not possible sufficient conditions. But on the other hand it is mutual knowledge between the speaker and the hearer that there are other sufficient conditions for the truth of the consequent, such as cleaning the streets with water, a broken pipe, etc. Attributing to the speaker the belief that these are not sufficient conditions conflicts with knowing that the speaker believes they are. This yields the conclusion that the speaker tries to convey something she knows to be false, and so that she is not truthful. A conflict occurs between the assumption that the speaker observes the first submaxim of Quantity (which is essential in deriving the scalar implicature) and the assumption that she observes the first submaxim of Quality (Do not say what you believe to be false). The assumption that the speaker observes one maxim took us to conclude that she violates a different one, which has a special status. Grice suggests that it might be true that "other maxims come into operation only on the assumption that this maxim of Quality is satisfied" (Grice 1989. p. 27). If indeed the first submaxim of Quality has this special status in the hierarchy of maxims, the attempt to perfect the conditional is ultimately illegitimate in this case.

So, there are cases of DA argument-w's in which the conditional cannot be perfected, and so no implicature can be derived that would lead to a valid argument-m. There might also be more complicated cases in which the implicature is not clearly derivable, or in which the assumption that the speaker observes the maxim of Quantity involved in deriving the implicature would result in a conflict with the assumptions that she is observing other maxims, which do not have such a special status in the hierarchy as the first submaxim of Quality. ${ }^{11}$ The complicated problem, which I will not be able to solve here, is to determine in which contexts the scalar implicature is licensed and in which it is not.

Third, a general comment about the interaction between CP and DA arguments. Horn talks about "the fallacy of perfection"

\footnotetext{
${ }^{11}$ According to Geis and Zwicky only the maxim of Quality could block the scalar implicature: "conditionals are understood to be perfected unless the hearer has reason to believe that the converse is false." (Geis\&Zwicky 1971 p.5)
} 
(idem, p. 317) 12 and about "the analysis of CP and its family of fallacies" (idem p. 304). I want to point out that CP cannot explain the psychological tendency to perfect conditionals, and so it cannot be an explanation of why people commit these fallacies. In general, Gricean accounts of implicatures are not psychological hypotheses, so they are not explanations of tendencies people may have to take an utterance as conveying more than it expresses in virtue of its conventional meaning. As Kent Bach writes:

Grice did not intend his account of how implicatures are recognized as a psychological theory or even as a cognitive model. He intended it as a rational reconstruction. When he illustrated the ingredients involved in recognizing an implicature, he was enumerating the sorts of information that a hearer needs to take into account, at least intuitively, and exhibiting how this information is logically organized. He was not foolishly engaged in psychological speculation about the nature of or even the temporal sequence of the cognitive processes that implements that logic. (Bach 2006, p. 8.)

Gricean rational reconstruction are appropriate for some cases in which people treat sentences of the form 'if $p, q$ ' as expressing biconditionals; more precisely, of those cases in which a scalar implicature is present. They are not reconstructions of all cases of CP because not all of them are rational (i.e., justified) inversions of the conditional. The scalar implicature is not present in all cases in which there is a tendency of the audience to take a conditional as expressing a bi-conditional. In general, a Gricean account of implicature is compatible with there being cases in which the audience takes the speaker to have implicated something, but there is no implicature. ${ }^{13}$ Similarly, a Gricean account of $\mathrm{CP}$ is

\footnotetext{
${ }^{12} \mathrm{He}$ also writes that, "Aristotle's presentation of the fallacy of consequence as an error in conversion renders this fallacy in a form quite closely akin to CP." (Horn 2000 p.298)

${ }^{13}$ For the purposes of this paper I will not follow the tradition of interpreting Grice as claiming that conversational implicatures forms part of what the speaker actually intends to convey, i.e., actually means. This interpretation is to be found in Neale (1992 p.14). However, according to Saul (2002) this interpretation is not correct. In particular, what is implicated does not depend on the speaker's actual intentions. That is, the speaker may implicate that $q$ by saying $p$ even if she does not intend to convey $q$. This interpretation of Grice is required by the assumption that the speaker is rational and observes the maxims of cooperative behaviour. At times speakers may fall short of these requirements, but this does not influence the rational reconstruction of the speaker's intentions, even if the reconstructed intentions do not coincide with the actual ones. Reference to speaker's intentions is here to be understood as reference to speaker's rationally reconstructed intentions and not actual intentions. The same thing with respect to speaker meaning: it is not what the speaker actually means,
} 
compatible with there being cases in which we treat the conditional as a bi-conditional, but no scalar implicature is actually present. A Gricean account of $\mathrm{CP}$ is not concerned with the tendency to (intuitively) perfect conditionals, but with giving a reconstruction of those cases in which it is correct to do so. The above reconstructions explain our tendency as speakers or hearers to perfect conditionals only in those cases in which this tendency is rational, and so can be rationally reconstructed. But sometimes intuitions are not reliable. Not in all cases in which we have the intuition that the utterance of a conditional expresses a biconditional does it really do so. As several authors have pointed out, people tend to perfect the conditional especially in cases of promises, threats, warnings, prohibitions or commands, and it seems that in such cases, such as (1), the derivation of the scalar implicature is correct, so the tendency to perfect the conditional is justified. But sometimes people perfect the conditional when they should not: "Ever since Aristotle pinpointed the temptation to infer If the streets are wet, it has rained and If he's hot, he has a fever, however, it has also been clear that the conversion or perfection of conditionals cannot be restricted to warnings, threats, or promises" (Horn 2000, p. 319).

These observations help deal with a possible objection to my account of (1) and other similar cases. Jonathan Adler suggests a different interpretation of this case:

[A]n obvious alternative to viewing the child as fallaciously reversing a conditional is that the child treats his conditional as really a bi-conditional. My claim is that there need be no rivalry between the view that the child meant his conditional as a bi-conditional, and that his reasoning involved a fallacious reversal of the conditional. For the child's meaning by that conditional a biconditional, is itself plausibly due to his treating the conditional as reversible. (Adler 1994, p. 227)

Adler suggests that the child meant by his conditional a biconditional because "his reasoning involved a fallacious reversal of the conditional". If the reversal is fallacious, it cannot be due the intention to convey a scalar implicature, because in the case of an implicature the child has reasons to think the sufficient condition is also necessary, and so the reversal of the conditional is not fallacious. What Adler suggests is that the child reverses the conditional with no reason. This is due possibly to a confusion between the meanings of 'if $p$ then $q$ ' and 'if $q$ then $p$ ', or to the

but what a rational and cooperative speaker would mean by the sentence uttered in the given context. 
false belief that the latter follows from the former. In any case it is due to a confusion; or at least this is what Adler seems to suggest. ${ }^{14}$ What I have argued above is that in cases such as (1), in which the argument-w may be a DA, the argument-m is not of DA form, but is modus ponens, given that the scalar implicature is a premise in the argument. But the objection is that there is no implicature involved, but merely confusion about what the first sentence in (1) expresses. It is her confusion that explains her reversing the conditional, not the reasoning involved in scalar implicatures. ${ }^{15}$

What needs to be said in reply to this objection is that the child's treatment of a conditional as a bi-conditional may indeed be explained as some sort of a confusion. But, on the interpretation of Gricean rational reconstructions that I endorse, it does not follow from this that no implicature is present. An implicature is expressed, and so is the argument-m, which is not of DA form; this is true independently of whether the speaker or her audience realizes it in the context of the utterance. The presence of the implicature, and consequently, of the argument-m, does not depend on the speaker's actual intentions, or on the intentions that a given audience may attribute to her; it is a matter of what an utterance of a sentence expresses in a context to a rational audience; and it is recovered through rational reconstructions by way of Gricean derivations, and not through psychological investigations of a speaker's actual intentions. It may be that a speaker is not aware of what her utterance implicates in the context; and that the actual audience does not recover the scalar implicature either; but that does not mean that no implicature is expressed. The derivation of the scalar implicature according to the schema mentioned above does not depend in any way on the speaker's actual intentions. However, if the speaker is not aware of the derivation, then she may have treated the conditional as reversible for other reasons, and maybe for no good reason at all, but due to confusion. Some error has been committed in that case. But still, a valid argument-m has been expressed, and a rational audience is able to reconstruct it.

\footnotetext{
${ }^{14}$ The idea that there is a confusion involved in such cases comes up in the writings of other authors as well: "Perhaps we tend to confuse If A, then B with If $\mathrm{B}$, then $\mathrm{A}$ because if $\mathrm{B}$ follows from $\mathrm{A}$, it is fairly common for $\mathrm{A}$ to follow from B also" (Cederblom \& Paulsen 2006, p. 165, my emphasis). Also C. Tindale: "It is clear that if we have one form that is valid and another that is very similar to it but invalid, then someone could confuse the two. That is why formal fallacies are sometimes called fallacies of resemblance" (Tindale 2007, p. 50, my emphasis).

${ }_{15}$ Adler's words suggest this line of reasoning. However, even if my interpretation of his words is incorrect, this objection remains a possible one and needs to be dealt with.
} 


\section{Acknowledgements}

The investigation for this paper was made possible by the support of a Spanish FPI grant associated to the Research Project HUM200608236. I am especially grateful for helpful comments to Robert Pinto and David Godden.

\section{References}

Adler Jonathan. (2002). Belief's Own Ethics. Cambridge, MA: MIT Press.

Adler, Jonathan (1994) Fallacies and alternative interpretations. Australasian Journal of Philosophy, 72:3, 271-282.

Auwera, Johan van der. (1997). Pragmatics in the last quarter century: The case of conditional perfection. Journal of Pragmatics, 27, 261-274

Bach, Kent. (2006). The Top 10 misconceptions about implicature. In B. Birner \& G. Ward (Eds.), A Festschrift for Larry Horn. Amsterdam: John Benjamins.

Burke, Michael B. (1994). Denying the antecedent: A common fallacy? Informal Logic, 16(1), 23-30.

Cederblom, Jerry \& David W. Paulsen. (2006). Critical Reasoning, $6^{\text {th }}$ ed. Belmont, CA: Wadsworth Publishing Company.

Ducrot, Oswald. (1969). Présupposés et sous-entendus. Reprinted in: O. Ducrot, Le dire et le dit, pp. 13-31. Paris: Les Editions de Minuit, 1984.

Johnson, Ralph H. (2000). Manifest Rationality: A Pragmatic Study of Argument, Mahwah, NJ: Lawrence Erlbaum Associates.

Hitchcock, David. (1995). Did Jesus commit a fallacy? Informal Logic, 17(2), 297-302.

Horn, Laurence R. (2000). From if to iff: Conditional perfection as pragmatic strengthening. Journal of Pragmatics 32, 289-326.

Geis, Michael L. \& Arnold M. Zwicky. (1971). On invited inferences. Linguistic Inquiry 2, 561-566.

Godden, David \& Douglas Walton. (2004). Denying the antecedent as a legitimate argumentative strategy: A dialectical model. Informal Logic, 24(3), 219-243.

Grice, H.P. (1989). Studies in the Way of Words. Cambridge, MA: Harvard University Press.

Krabbe, E.C.W. (2003). The pragmatics of deductive arguments. The Proceedings of the OSSA Conference: InformalLogic@ 25. Windsor, ON: CD ROM.

Neale, Stephen (1990) Descriptions. Cambridge: MIT Press Books.

Neale, Stephen (1992) Paul Grice and the philosophy of language. Linguistics and Philosophy, 15, 509-559. 
326 Andrei Moldovan

Saul, J.M. (2002). Speaker meaning, what is said and what is implicated. Nous, 36 (2), 228-248.

Pinto, Robert C. (2001). Argument, Inference and Dialectic. Dordrecht: Kluwer

Tindale, Christopher W. (2007). Fallacies and Argument Appraisal. Cambridge: Cambridge University Press.

Wilson, D., and Sperber, D. (1986). Inference and implicature. In S. Davis (Ed.). Pragmatics: A Reader, pp. 377-393. Oxford: Oxford University Press, 1991. 